\title{
Comparison of Numerical Simulations of the Electron Density in the D-Region lonosphere with Measurements of the Radar of Partial Reflections
}

\author{
Aleksandr Gomonov ${ }^{1,2, *}$, Roman Yurik ${ }^{1,2}$, Yulia Shapovalova ${ }^{1}$, Sergei Cherniakov, ${ }^{1}$ and Olga Ogloblina ${ }^{1}$ \\ ${ }^{1}$ Polar Geophysical Institute, RU-183010, Murmansk, Russian \\ ${ }^{2}$ Murmansk Arctic State University, RU-183038, Murmansk, Russian Federation
}

\begin{abstract}
The paper reports results of a comparison of the measured electron density in the ionospheric Dregion measured using the partial reflection facility at the observatory. Tumanny of the Polar Geophysical Institute $\left(69.0^{\circ} \mathrm{N}, 35.7^{\circ} \mathrm{E}\right)$ with numerical simulations performed using the theoretical model of the Polar Geophysical Institute (PGI) (Murmansk, Russian Federation). The model was examined using experimental data obtained under quiet geomagnetic conditions in March, 2017. The comparative analysis carried out in this study shows a very good agreement of the PGI model with experimental data and indicates that the IRI2016 model fails to adequately reproduce measurements in regions with high electron density gradients.
\end{abstract}

\section{Introduction}

The $D$-region of the ionosphere covers the altitude range of $50-90 \mathrm{~km}$. The knowledge of its properties is very important as well as for applied interest and for scientific one. The $D$-region of the ionosphere has a significant influence on the radio waves propagation in the wide frequency bands. The $D$-region remains not enough studied region of the ionosphere for various reasons. The behavior of the $D$-region of the ionosphere is quite complicated even in calm conditions of mid-latitudes, and is quite difficult for experimental study because there are the low electron density, the complex composition of the ions and the high density of the neutral atmosphere. The behavior of the ionosphere at high latitudes is much more complex, as corpuscular sources of ionization are very often observed: mainly electrons in the auroral oval and solar protons in the polar region. It is very impotent to create mathematical, and then computer models of the $D$-region that can describe the behavior of the main ionospheric parameter - the electron density $\left(N_{e}\right)$ - under various heliogeophysical conditions.

The radar has been designed by the Polar Geophysical Institute (PGI) (Murmansk, Russian Federation) to study the lower ionosphere by the method of partial reflections [1-3] and have been used for investigation and comparisons with methods [4-5]. The medium frequency (MF) radar of vertical sounding of the ionosphere by the partial reflection method is the main effective instrument for the quantitative study of the lower ionosphere, which allows continuous observations for a long time. This radar is situated at the radio physical observatory Tumanny of the Polar
Geophysical Institute (69.0 N, 35.7 E) and is unique one. It has been working since 1991 on a regular basis.

\section{Characteristics of the medium frequency radar of vertical sounding}

The characteristics of the medium frequency radar for vertical sounding of the ionosphere are represented in Table 1.

Table 1. Technical characteristic of the MF-radar.

\begin{tabular}{|c|c|}
\hline Transmitter & Receiver \\
\hline $\begin{array}{c}\text { Power per pulse at } \\
2.6 \mathrm{MHz} \sim 100 \mathrm{~kW} .\end{array}$ & Sensitivity $-0.5 \mu \mathrm{V}$ \\
\hline Pulse duration - $15 \mu \mathrm{s}$ & Bandwidth $-50-60 \mathrm{kHz}$ \\
\hline $\begin{array}{c}\text { o- and x- waves repetition } \\
\text { frequency - } 1 \mathrm{~Hz}\end{array}$ & Height range - 30-240 km \\
\hline $\begin{array}{c}\text { Delay between the pulses } \\
275 \text { ms. }\end{array}$ & $\begin{array}{c}\text { Registration range - any } \\
\text { within the height range. } \\
\text { The height step is } 0.5 \cdot n \mathrm{~km} \\
(n=1,2,3, \ldots) .\end{array}$ \\
\hline
\end{tabular}

The antenna array consists of $32(4 \times 8)$ extended range cross-vibrators with a rotating field polarization (Fig. 1). This antenna array available for transmission and receiving electromagnetic waves of the ordinary and extraordinary polarization in dependence of the operation mode. The antenna array occupies an area of $55000 \mathrm{~m}^{2}$, and has a gain about 12.5-18.7 dB. The antenna field has longer side in the direction from south- 
west to north-east. Therefore, the beam width is nonsymmetrical and it equal to $19^{\circ}$ along the north-east direction and $30^{\circ}$ along the north-west direction. The side lobe level is $12 \%$ [6].

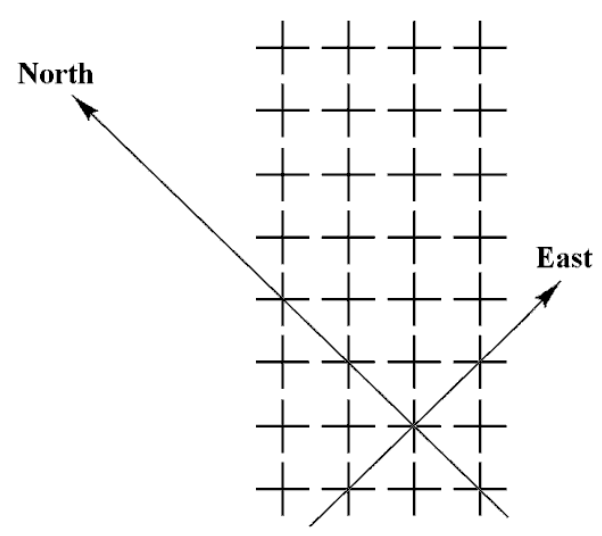

Fig.1. The MF-radar antenna array layout.

\section{Measurements}

It is known that electron density in the D-region of the ionosphere can be obtained by calculations from the measured amplitudes of ordinary $A_{0}(h)$ and extraordinary $A_{\mathrm{x}}(h)$ waves partially reflected by the ionospheric plasma in two ways: by the differential absorption method or by the correlation method.

These methods for determination plasma parameters are based on the emission of two wave modes in the form of alternating pulses. Separate receiving of the signals partially reflected by electron density irregularities and suggested frequency of collisions between electrons and neutral particles, as well as measured amplitudes in dependence on the time delay determined by the reflection height constitute this method $[7,8]$.

The distribution of electron density $N_{e}(h)$ (Fig. 2) can be determined from the measured ratio $A_{\mathrm{x}}(h) / A_{\mathrm{o}}(h)$, under certain conditions, using the equation:

$$
N(h)=\frac{\frac{d}{d h}\left[\ln \left(R(h)-\ln \frac{A_{x}(h)}{A_{o}(h)}\right)\right]}{K(h)},
$$

where $K(h)=2\left[k_{\mathrm{x}}(h)-k_{\mathrm{o}}(h)\right] / N, \quad k_{\mathrm{x}, \mathrm{o}}(h) \quad$ - are the absorption coefficients of the extraordinary (x) and ordinary (o) radio waves, determined by the operating frequency, the electron gyrofrequency and the altitude profile of the electron-molecule collisions frequency, $R(h)=\left|R_{\mathrm{x}}(h)\right| /\left|R_{\mathrm{o}}(h)\right|, \mid R_{\mathrm{x}}$ o $(h) \mid$ - are the absolute values of reflection coefficients.

Measurements of the electron density of $N_{e}$ in the $D-$ region of the ionosphere are both expensive and local in space. In this regard, a computer model of the ionizationrecombination cycle of the $D$-region of the ionosphere was created at the Polar Geophysical Institute (Murmansk) [9].

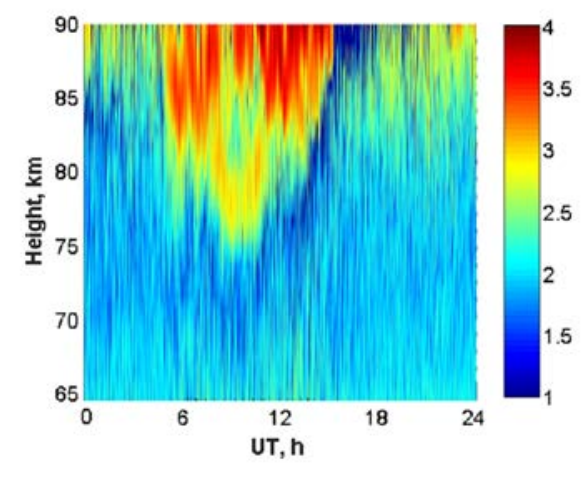

Fig. 2. An example of measuring of the electron density $\operatorname{Lg}\left(N_{e}\right)\left(\mathrm{cm}^{-3}\right) 13$ March 2017.

This model includes four positive ions $\mathrm{NO}^{+}, \mathrm{O}_{2}{ }^{+}$, $\mathrm{Cb}_{1}{ }^{+}, \mathrm{Cb}_{2}{ }^{+}$and four negative ions $\mathrm{O}_{2}^{-}, \mathrm{O}^{-}, \mathrm{CO}_{3}{ }^{-}, \mathrm{NO}_{3}{ }^{-}$ and electrons [9]. The justification of the model, the constants of the velocities of processes in it are given in. The model takes into account the variations of $N_{e}(h)$ due to the zenith angle of the Sun, solar activity, and the seasons of the year. The profile $N_{e}(h)$ in the model is determined by the following analytical expression:

$N e(h)=N_{0}(h) \cos ^{n(h)} \chi_{k p}\left[1+k(h) \cos \left(\frac{m-91}{365} 360^{0}\right)\right] e^{\alpha(h) R}$

where $N_{0}(h)$ - is the electron density profile corresponding to the day of the autumn (spring) equinox at the effective zenith angle of the sun $\chi_{k p}=0$ and the minimum solar activity $R=0$.

The exponent in formula (2), which determines the dependence of $N_{e}(h)$ on the zenith angle of the Sun, is represented by the function:

$$
n(h)=A_{n} \cos \left(\frac{B_{n}-h}{C_{n}} 360^{0}\right)
$$

with coefficients $A_{n}=0,9, B_{n}=80,0, C_{n}=131,2$.

This gives a maximum dependence of $N_{e}(h)$ on $\chi_{k p}$ at $80 \mathrm{~km} \cos ^{0,9} \chi_{k p}$ and a minimum $\cos ^{0,5} \chi_{k p}$ at the height of $50 \mathrm{~km}$.

The corrected (effective) zenith angle of the sun had been used in order to take into account asymmetry of daily variations $\chi_{k p}(t)=\chi(t-0,5)$, where $t$ - local time in hours.

The dependence of the electron density on solar activity (the number of sunspots $R$ ) is given by the ratio:

$$
N e \sim e^{\alpha(h) R}
$$

The proportionality coefficient $\alpha(h)$ (determined only from rocket data) is approximated by the expression:

$$
\alpha(h)=A_{\alpha}\left(1-\frac{C_{\alpha}}{h-B_{\alpha}}\right)
$$

with parameters $A_{\alpha}=0,015, C_{\alpha}=1,33, B_{\alpha}=50,0$. 
Electron density decreases when increasing solar activity at the height of $60 \mathrm{~km}$ and grows in the heights range of $62-90 \mathrm{~km}$ in accordance with (5). The growth of $N_{e}(h)$ becomes sharper with increasing of height. The electron density $N_{e}(h)$ increases in 1.9 times at the height of $70 \mathrm{~km}$ and 2.9 times at the height of $90 \mathrm{~km}$ when passing from a minimum $(R=0)$ to a maximum of solar activity.

It had been shown earlier [9] that this model allows to reliably describe seasonal variations of the ionic composition and electron density, which are reliably established from rocket data, as well as the winter anomaly of the region $D$.

The calculation of the model (PGI) provides the scientists and researchers with a number of options that allow solving various problems. This model is the most suitable for estimation of the electron density in the $D$ region in the Arctic region.

Comparison analysis between of experimental data of measurements with the model calculations was carried out for selected dates under quiet geomagnetic conditions (March 13, 14, 16-20, 24-26, 2017) (Fig. 3, 4).

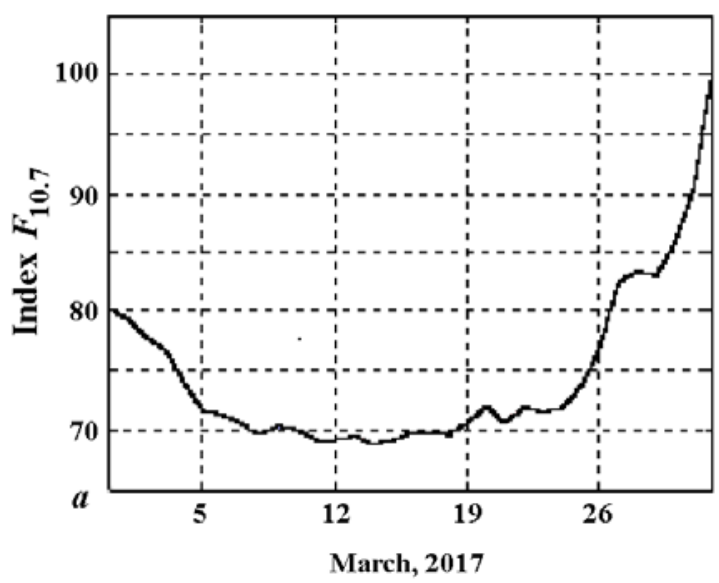

Fig. 3. $F_{10.7}$ index (solar radiation flux at the wavelength of $10.7 \mathrm{~cm}$, in units $\times 10^{22} \mathrm{~W} /\left(\mathrm{m}^{2} \cdot \mathrm{Hz}\right)$.

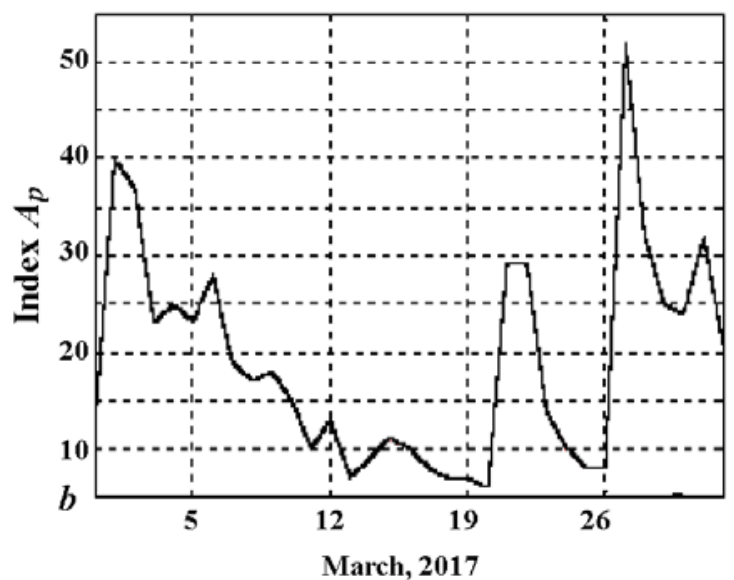

Fig. 4. $A_{p}$-index.

As it can be seen from the diagram in Fig. 3, during the period from March 13 to March 26, the flux of solar radio emission at the wavelength of $10.7 \mathrm{~cm}$ (index $F_{10.7}$, left panel) varied slightly, within 68.8-76.8 (in units of $\left.10^{22} \mathrm{~W} /\left(\mathrm{m}^{2} \cdot \mathrm{Hz}\right)\right)$. For this period, the $A_{p}$-index in Fig. 4 ranged from 1 to 24 , which corresponds to the $K_{p}$ values from $0+$ to $4-$.

The measurement data of $N_{e}(h)$ were compared with the simulation results on selected days. The coordinates of the partial reflection radar were set in the (PGI) model. The data of solar activity parameters were fed to the model input. The averaged measurement data were compared with the model averaged data.

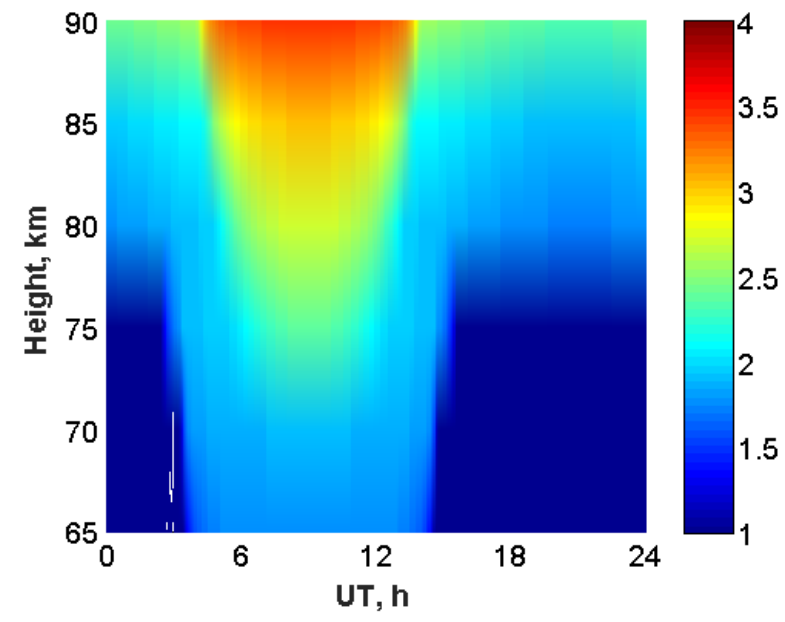

Fig. 5. The average electron density of model data $\left(\lg \left[N_{e}\right]\right.$, $\mathrm{cm}^{3}$ ) on March 13, 14, 16-20, 24-26, 2017.

In the Fig.5 diurnal variations of electron density profiles are shown in the altitudinal range from 65 to $90 \mathrm{~km}$ according to model calculations and observations in Fig.6.

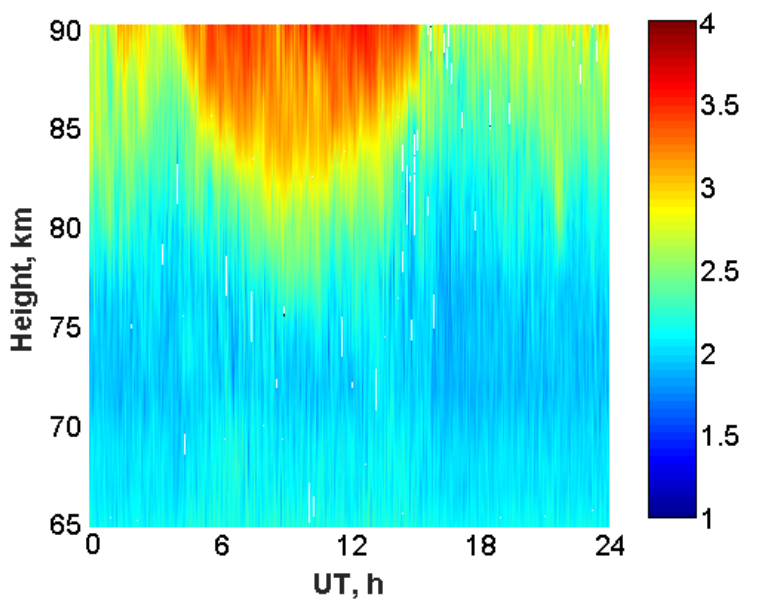

Fig. 6. The averaged electron density of experimental data $\left(\lg \left[N_{e}\right], \mathrm{cm}^{-3}\right)$ on March 13, 14, 16-20, 24-26, 2017.

The ten-days averaged electron density profiles $N e(h)$ were constructed from the model and observations data at the four moments: 02:00 UT, 10:00 UT, 12:00 UT, and 22:00 UT and are shown in Fig. 7 (red lines correspond to the calculations by model (PGI) and blue lines correspond to the profiles calculated from measurements).

It can be seen from the above diagrams (Fig. 7) that the PGI model quite well describes the electron density 
profiles of $N_{e}(h)$ on days with a quiet geomagnetic situation for different times of the day.
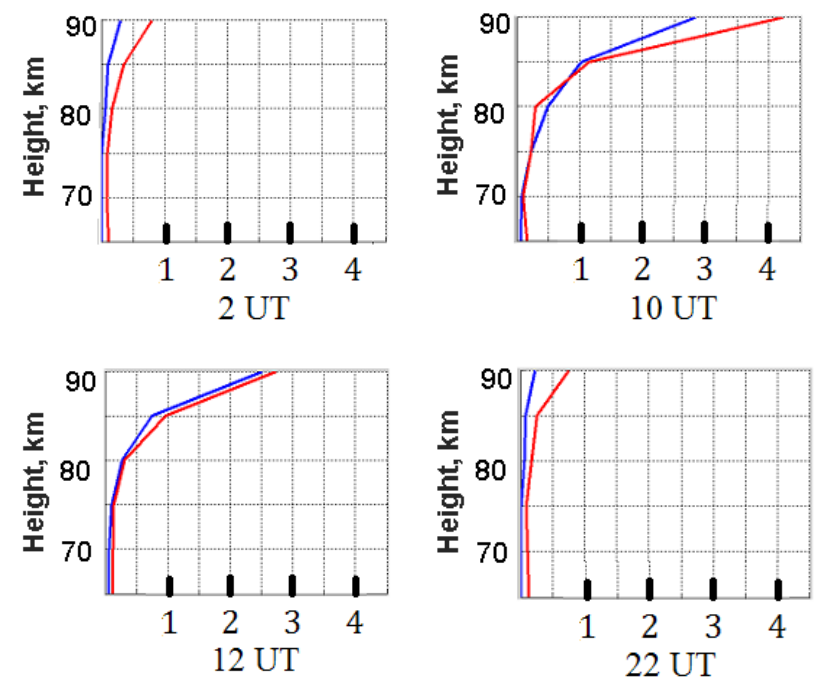

Fig. 7. Electron density $\left(\lg \left[N_{e}\right], \mathrm{cm}^{-3}\right)$.

\section{Conclusions}

The algorithm for calculating of the electron density which was presented in this article and the computer model (PGI) was created on its basis are based on modern concepts of the ionization-recombination cycle in the lower ionosphere. The model takes into account all the main sources of ionization in the $D$-region of the ionosphere under quiet geomagnetic conditions. The ion-molecular reactions are included in the model make it possible to describe the influence of a neutral atmosphere, its daily and seasonal variations on ionospheric parameters (electron density). The model allows to take into account the heights variations of electron density, its seasonal changes for quiet geomagnetic conditions.

The calculation of the relative errors of measurements of the electron density of $N_{e}(h)$ with model data is shown in (Fig. 8).

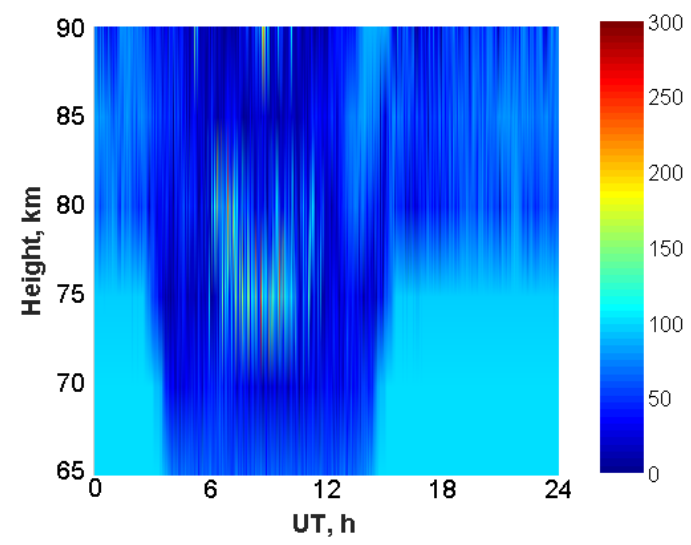

Fig. 8. The calculated relative errors of $\mathrm{Ne}(\mathrm{h})$ determination.

Relative errors were calculated as a ratio between module of difference experiment data and model data of electron density to the module of experiment data of electron density. Then the obtained result was multiplyed on 100.

The standard deviation of the measurement data of electron density is represented in Fig. 9.

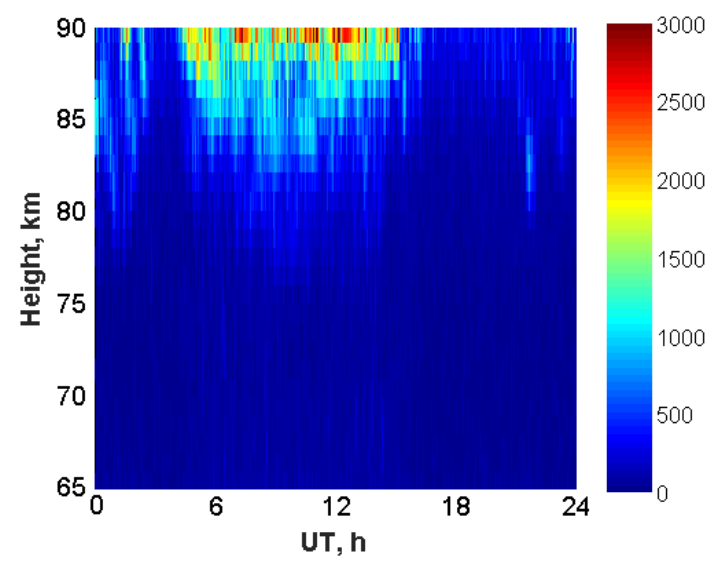

Fig. 9. The standard deviation of the measurement data of electron density.

As we can see in Fig. 9 the standard deviation of the measurement data of electron density for days for quiet geomagnetic conditions have not essential variations except for measurements on high heights in day time. This fact is obvious.

As can be seen (Fig. 8), the (PGI) model can be considered as adequate one, and the results of its simulation $N_{e}(h)$ for the $D$-region of the ionosphere are consistent with the measurement data. This can be explained by the fact that the (PGI) model of electron density was specially developed for the Arctic region, and its parameters were selected for the conditions of the high latitudes. On the contrary of the (PGI) model, the IRI-2016 model, should be adjusted in the areas with high gradients of electron density $N_{e}(h)$ [10].

\section{References}

1. V.A. Tereshchenko, V.D. Tereshchenko, S.M. Chernyakov, Proc. MSTU 13(4/2), 1052-1059 (2010)

2. S.M. Chernyakov, V.A. Tereshchenko, O.F. Ogloblina, E.B. Vasiliev, A.D. Gomonov, Izv. vuzov. Fizika 12(2), 50-53 (Rus) (2016)

3. A.D. Gomonov, R.Yu. Yurik, Yu.A. Shapovalova, In. Science and Education in the Arctic Region 150155 (2018)

4. M. Klimenko, V. Klimenko, I. Despirak, I. Zakharenkova, B. Kozelov, S. Cherniakov, E. Andreeva, E. Tereshchenko, A. Vesnin, N. Korenkova, A. Gomonov, E. Vasiliev, K. Ratovsky, Journal of Atmospheric and Solar-Terrestrial Physics 180, 78-92 (2018)

5. S.M. Cherniakov, V.A. Turyansky, A.D. Gomonov, Proc. SPIE 10833, 24th International Symposium on Atmospheric and Ocean Optic: Atmospheric Physics, 2-5 July 2018, Tomsk. 1083395.1 1083395.4 (13 December 2018) 
6. V.D. Tereshchenko, E.B. Vasiliev, N.A. Ovchinnikov, A.A. Popov, Technique and methods of geophysical experiments (KSC RAS, Apatity, 2003).

7. Ya.L. Alpert, Propagation of electromagnetic waves and the ionosphere (Nauka, Moscow, 1972).

8. J.A. Ratcliffe, An introduction to the physics of the ionosphere and magnetosphere (Cambridge University Press, Cambridge, 1972).

9. N.V. Smirnova, Modeling of the ionizationrecombination cycle of the D-region, in Mathematical modeling of complex processes (USSR Academy of Sciences, Apatity, 1982).

10. A.D. Gomonov, R.Yu. Yurik, Y.A. Shapovalova, S.M. Cherniakov, N.V. Kalitenkov, The analysis of electron density values according to simulations by IRI model and from experimental measurements by partial reflection radar, in IEEE Proceedings 8810341, 75-78 (2019). 